\title{
Handling the Handbag Diagram in Compton Scattering on the Proton
}

\author{
Gerald A. Miller \\ Department of Physics, University of Washington, Seattle, WA 98195-1560
}

(Dated: November 8, 2018)

\begin{abstract}
Poincaré invariance, gauge invariance, conservation of parity and time reversal invariance are respected in an impulse approximation evaluation of the handbag diagram. Proton wave functions, previously constrained by comparison with measured form factors, that incorporate the influence of quark transverse and orbital angular momentum (and the corresponding violation of proton helicity conservation) are used. Computed cross sections are found to be in reasonably good agreement with early measurements. The helicity correlation between the incident photon and outgoing proton, $K_{L L}$, is both large and positive at back angles. For photon laboratory energies of $\leq 6 \mathrm{GeV}$, we find that $K_{L L} \neq A_{L L}$, and $D_{L L} \neq 1$.
\end{abstract}

PACS numbers: $13.60 . \mathrm{Fz}$

Keywords: Compton Scattering, high momentum transfer, relativistic constituent quark models 


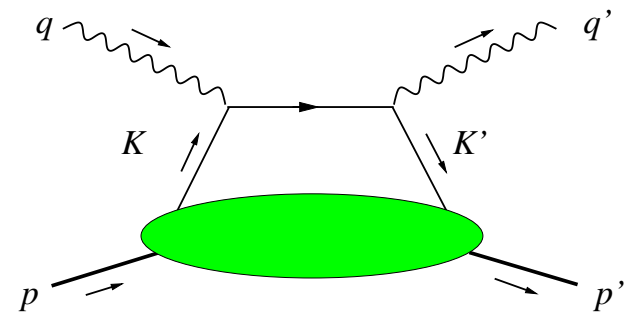

(a)

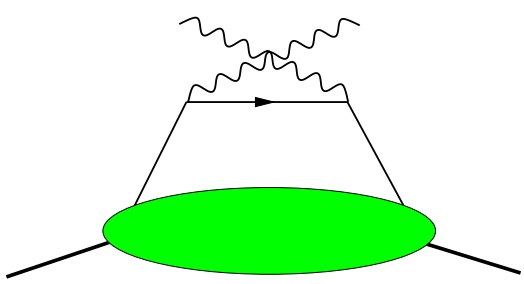

(b)

FIG. 1: (Color Online) Direct (a) and crossed (b) handbag graphs for the Compton amplitude.

The recent and planned experimental accessibility [1, 2] of Real Compton Scattering on the proton at large momentum transfer make this reaction a promising new probe of short distance structure. The scattering amplitude depends on the square of the charge of a struck quark, and so provides a ground-state to ground-state matrix element that is different than the ones involved in the electromagnetic form factors [3].

One primary goal has been to determine the dominant reaction mechanism that allows the proton to accommodate the large momentum transfer while remaining a proton. According to perturbative QCD (pQCD) [4, 5] the three active valence quarks share the momentum transfer via the exchange of two gluons that each carry a large momentum. In the pQCD treatment, hard gluon exchanges are included in an effective current operator, and so can be taken to occur within the time duration of the reaction. The most recent calculations 6] find that this mechanism yields cross sections that are about 10 times smaller than ones measured at photon energies of $6 \mathrm{GeV}$ or less. Another approach uses overlaps of soft non-perturbative wave functions [7, 8]. As noted in the review [9], the invariant amplitude is obtained by evaluating the so-called handbag diagrams of Fig. 11. The large momentum transfer occurs on a single quark, with a probability amplitude determined by the overlap of the initial and final state wave functions. Here the high momentum transfer is accommodated by the exchange of an uncountable number of gluons that occurs either before and after the reaction takes place. Such calculations have had reasonable success in reproducing the measurements [8, 10].

Previous treatments of the handbag diagram [7, 8] have provided a unifying relation between elastic form factors, real Compton scattering and virtual Compton scattering. This approach has led to interesting predictions that are testable experimentally. Our concern here is with understanding the limitations of the approximations. One involves asserting that the longitudinal momentum of the proton is carried by a single quark 8 . Another involves the neglect of the effects of hadron helicity flip [7, 8] in high momentum transfer exclusive reactions. A specific consequence is that the observables $K_{L L}$ (which involves the helicity of the final proton) and $A_{L L}$ (which involves the helicity of the initial proton) are predicted to be the same. Ref. [10] includes corrections that allow photon and proton helicity flip.

Our purpose here is to use a model wave function that provides a reasonably good description of all four nucleon electromagnetic form factors [11, 12, 13] to evaluate the graphs of Fig. 1 in a manner that avoids neglecting the effects of hadronic helicity non-conservation. The essential feature is that relativistic and quark mass effects induce significant quark transverse and orbital angular momentum that cause violations of hadronic helicity conser- 
TABLE I: Different parameter sets, $m, \beta$ in $\mathrm{fm}^{-1}$. From Ref. [13]

\begin{tabular}{|l|rrr|}
\hline Set(legend) & $m$ & $\beta$ & $\gamma$ \\
\hline 1 dash & 1.8 & 3.65 & 4.1 \\
2 dot-dash & 1.8 & 3.65 & 3.9 \\
3 solid & 1.7 & 2.65 & 3.7 \\
\hline
\end{tabular}

vation.

We begin describing the formalism by reviewing the salient features of the wave functions of Refs. [11, 12, 13]. This model starts with a wave function for 3 relativistic constituent quarks:

$$
\Psi\left(p_{i}\right)=u\left(p_{1}\right) u\left(p_{2}\right) u\left(p_{3}\right) \psi\left(p_{1}, p_{2}, p_{3}\right)
$$

where $p_{i}$ represents space, spin and isospin indices: $p_{i}=\mathbf{p}_{i} s_{i}, \tau_{i}$ and repeated indices are summed over. The spinors $u$ are canonical Dirac spinors. The components of momenta are expressed in terms of light cone notation: $\mathbf{p}_{i} \equiv\left(p^{+}, \mathbf{p}_{\perp}\right)_{i}$, with $p_{i}^{-}=\left(p_{\perp}^{2}+m^{2}\right) / p_{i}^{+}$. The three momenta $\mathbf{p}_{\mathbf{i}}$ of the quarks can be transformed to the total and relative momenta to facilitate the separation of the center of mass motion as $\mathbf{P}=\sum_{\mathbf{i}} \mathbf{p}_{\mathbf{i}}, \xi=\mathbf{p}_{\mathbf{1}}^{+} /\left(\mathbf{p}_{\mathbf{1}}^{+}+\mathbf{p}_{\mathbf{2}}^{+}\right), \eta=$ $\mathbf{p}_{\mathbf{1}}^{+}+\mathbf{p}_{\mathbf{2}}^{+} / \mathbf{P}^{+}, \mathbf{k}_{\perp}=(\mathbf{1}-\xi) \mathbf{p}_{\mathbf{1} \perp}-\xi \mathbf{p}_{\mathbf{2} \perp}, \mathbf{K}_{\perp}=(\mathbf{1}-\eta)\left(\mathbf{p}_{\mathbf{1} \perp}+\mathbf{p}_{\mathbf{2} \perp}\right)-\eta \mathbf{p}_{\mathbf{3} \perp}$. One may express the proton wave function in the center of mass frame in which the individual momenta are given by $\mathbf{p}_{1 \perp}=\mathbf{k}_{\perp}+\xi \mathbf{K}_{\perp}, \mathbf{p}_{2 \perp}=-\mathbf{k}_{\perp}+(1-\xi) \mathbf{K}_{\perp}, \mathbf{p}_{3 \perp}=-\mathbf{K}_{\perp}$. The structure of the color-spin-isospin wave function can be understood in a familiar form. This eigenstate of spin [14, 15] is a product of an anti-symmetric color wave function with a symmetric flavorspin-momentum wave function, given by $\Psi=\frac{1}{\sqrt{2}}\left(\phi_{\rho} \chi_{\rho}+\phi_{\lambda} \chi_{\lambda}\right) \Phi$, where $\phi_{\rho}$ represents a mixed-antisymmetric and $\phi_{\lambda}$ a mixed-symmetric flavor wave function and, $\chi_{\rho, \lambda}$ represents mixed symmetric or anti-symmetric spin wave functions in terms of Dirac spinors. The lower components of these contain terms in which the spin of the quark is opposite to that of the proton, with the difference accommodated by the orbital angular momentum. Such terms are responsible for reproducing the experimental feature that $Q F_{2} / F_{1}$ is approximately constant for $Q^{2} \geq 2(\mathrm{GeV} / \mathrm{c})^{2}[11,12,13]$.

The spin-independent momentum-space wave function is a function of the mass operator $M_{0}$ of a non-interacting system of any $P^{\mu}: M_{0}^{2}=\left(K_{\perp}^{2}+m^{2} \eta\right) / \eta(1-\eta)+$ $\left(k_{\perp}^{2}+m^{2}\right) /(\eta \xi(1-\xi))$, where $m$ is the $u, d$ quark mass. We take the $S$-state orbital function $\Phi\left(M_{0}\right)$ to be of a power law form: $\Phi\left(M_{0}\right)=N /\left(M_{0}^{2}+\beta^{2}\right)^{\gamma}$ that depends on $\beta$, $\gamma$ and the constituent quark mass $m$. Parameters of the Light Front Cloudy Bag Model[13], which makes the Cloudy Bag Model[16] relativistic, are displayed in Table I. The effects of the pion cloud are unimportant at high momentum transfer and are ignored here.

Using light front dynamics enables one to relate the proton wave functions in different reference frames with a kinematic boost. If the proton acquires a transverse momentum by the absorption of a quantity of momentum, $\boldsymbol{\Delta} \equiv\left(0, \boldsymbol{\Delta}_{\perp}\right)=\mathbf{q}-\mathbf{q}^{\prime}$ by a quark, the effects of the boost are obtained by replacing the momenta $\mathbf{k}_{\perp}, \mathbf{K}_{\perp}$ by $\mathbf{k}_{\perp}, \mathbf{K}_{\perp}-\eta \boldsymbol{\Delta}_{\perp}$.

The evaluation of the Compton scattering amplitude $\mathcal{M}_{S^{\prime}, S}\left(\boldsymbol{\epsilon}^{\prime}, \boldsymbol{\epsilon}\right)$ is made with an impulse approximation in which the Compton scattering occurs via the Born and crossed Born graphs of Fig. 1. The antisymmetric nature of $\Psi$ allows us to take the scattering to occur on the third quark of charge $Q_{3}$. The incident (outgoing) photon has four momentum and polarization vector $q, \boldsymbol{\epsilon}\left(q, \boldsymbol{\epsilon}^{\prime}\right)$, evaluated using the $\gamma p$ center of mass frame. $\mathcal{M}_{S^{\prime}, S}\left(\boldsymbol{\epsilon}^{\prime}, \boldsymbol{\epsilon}\right)$ depends on the 
initial spin $S$ and final spin $S^{\prime}$ of the proton, as well as on $\boldsymbol{\epsilon}, \boldsymbol{\epsilon}^{\prime}$ :

$$
\mathcal{M}_{S^{\prime}, S}\left(\boldsymbol{\epsilon}^{\prime}, \boldsymbol{\epsilon}\right)=3 Q_{3}^{2} \int d \eta d^{2} K_{\perp} \sum_{s, s^{\prime}} \rho_{S^{\prime}, s^{\prime} ; S, s}\left(\eta, \mathbf{K}^{\prime}, \mathbf{k}\right) \bar{u}\left(K^{\prime}, s^{\prime}\right) \mathcal{O}_{\boldsymbol{\epsilon}^{\prime}, \boldsymbol{\epsilon}}\left(K^{\prime}, K\right) u(K, s),
$$

where $\rho\left(\eta, \mathbf{K}^{\prime}, \mathbf{k}\right) \equiv \int d \xi d^{2} k_{\perp} \Psi_{S^{\prime}, s^{\prime}}^{\dagger}\left(\xi, \mathbf{k}_{\perp}, \eta, \mathbf{K}_{\perp}^{\prime}\right) \Psi_{S, s}\left(\xi, \mathbf{k}_{\perp}, \eta, \mathbf{K}_{\perp}\right)$, and in which the repeated indices $\left(s, s^{\prime}\right)$ represent both spin and isospin (charge) quantum numbers of the struck third quark. The quantities $K, K^{\prime}$ are four vectors given by $K^{\mu}=\left((1-\eta) M_{0},-\mathbf{K}\right)$ and $K u(K, s)=m u(K, s), K^{\prime} u\left(K^{\prime}, s\right)=m u\left(K^{\prime}, s\right)$. Satisfying the latter two relations is necessary to maintain gauge invariance. The operator $\mathcal{O}_{\boldsymbol{\epsilon}^{\prime}, \boldsymbol{\epsilon}}\left(K^{\prime}, K\right)$ represents Compton scattering on a quark:

$$
\mathcal{O}_{\boldsymbol{\epsilon}^{\prime}, \boldsymbol{\epsilon}}\left(K^{\prime}, K\right)=\phi^{\prime *} \frac{\frac{1}{2}\left(K+q+K^{\prime}+q^{\prime}\right) \cdot \gamma+m}{(K+q)^{2}-m^{2}} \notin+\phi_{\frac{1}{2}\left(K^{\prime}-q+K-q^{\prime}\right) \cdot \gamma+m}^{\left(K^{\prime}-q\right)^{2}-m^{2}} \phi^{\prime *} .
$$

The numerators appearing in Eq. (3) are displayed in a form symmetric with respect to the initial and final states. This is necessary to maintain the time reversal invariance of the resulting amplitudes. Deriving Eq. (2) from the full four-dimensional formalism involves integration over the minus components of the wave function and neglecting modifications of the intermediate propagator of the struck quark caused by spectator quarks. Note also that the total momentum of the proton does not enter into the expression (2), so that our results are independent of frame.

Each of $s, s^{\prime}, \boldsymbol{\epsilon}, \boldsymbol{\epsilon}^{\prime}$, has two possible values, so there are 16 amplitudes. Using parity conservation and time reversal invariance reduces the number of independent amplitudes to six [17]. We calculate the 16 amplitudes and demonstrate explicitly that there really are only six independent ones. It is not obvious that the impulse approximation used in obtaining Eq. (2) will yield only six amplitudes. Applying parity invariance immediately reduces the number of independent amplitudes to eight. But the effects of time reversal invariance are more difficult to satisfy. This is because the sum of the quark minus-momenta is not equal to the minus-momentum of the proton. Thus conservation of four-momentum occurs only at the hadronic level, but not in the $\gamma q$ scattering. However, numerical calculations show that a reasonably accurate approximation can be made that leads to the respect of time reversal invariance. Examine Eq. (2) and shift the variable of integration according to $\mathbf{K}_{\perp} \rightarrow \mathbf{K}_{\perp}+\eta \boldsymbol{\Delta} / 2$. Then note that if the momentum transfer $\Delta$ is large compared to typical momenta appearing in the wave function, one may ignore the component of $\mathbf{K}$ parallel to $\boldsymbol{\Delta}$ in evaluating the matrix element $\bar{u}\left(K^{\prime}, s^{\prime}\right) \mathcal{O}\left(\boldsymbol{\epsilon}^{\prime}, \boldsymbol{\epsilon}\right) u(K, s)$. Numerical work shows that using this approximation doesn't change the computed values of observables by significant amounts, but does reduce the number of independent amplitudes to exactly six, and also maintains gauge invariance.

The relevant experimental observables involve photons and protons of a definite helicity. The $\gamma p$-cm helicity amplitudes are determined by making a unitary transformation on the spin amplitudes $\mathcal{M}_{S^{\prime}, S}\left(\boldsymbol{\epsilon}^{\prime}, \boldsymbol{\epsilon}\right)$ :

$$
\Phi_{\mu^{\prime} \lambda^{\prime}, \mu \lambda}=\sum_{S^{\prime}, S} T^{*}\left(\mathbf{p}^{\prime}\right)_{S^{\prime} \lambda^{\prime}} \mathcal{M}_{S^{\prime}, S}\left(\boldsymbol{\epsilon}_{\mu}^{\prime}, \boldsymbol{\epsilon}_{\mu}\right) T_{S, \lambda}(\mathbf{p}),
$$

where $\mu, \lambda$ represent the helicity of the initial photon and initial proton, and $T_{S, \lambda}(\mathbf{p}) \equiv$ $\bar{u}(p, s) u_{H}(p, \lambda) /\left(2 M_{p}\right)$ in which $u$ represents an ordinary Dirac spinor and $u_{H}$ represents a 


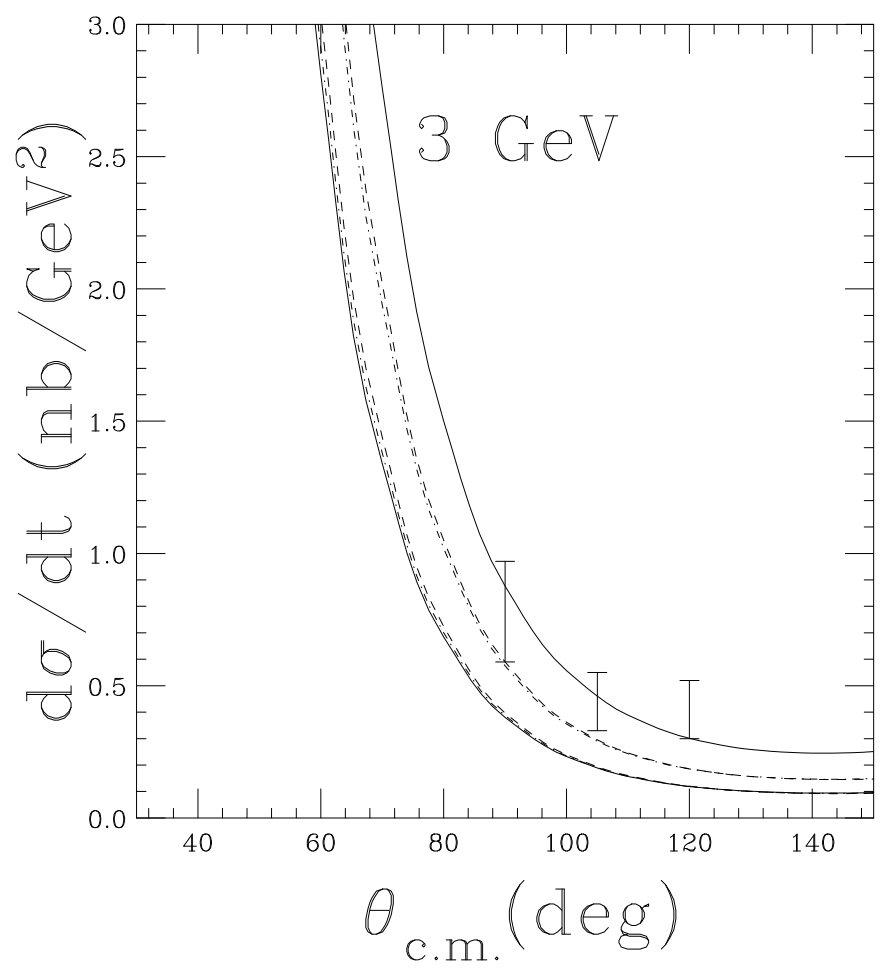

FIG. 2: Cross sections. The data are from Ref. 18]. Three curves are obtained with parameters defined in Table 1 and three are obtained by increasing the quark mass $m$ by $10 \%$.

helicity spinor. Then the differential cross section is expressed as:

$$
\frac{d \sigma}{d t}=\frac{1}{64 \pi\left(s-m^{2}\right)^{2}} \Sigma_{\mu, \mu^{\prime}, \lambda, \lambda^{\prime}}\left|\Phi_{\mu^{\prime}, \lambda^{\prime}, \mu \lambda}\right|^{2}
$$

Our results for this are shown in Fig. 2. Using the parameter set of Table I leads to cross sections that are a bit too small, but increasing the quark mass by $10 \%$, leads to qualitatively good agreement. The dependence of $d \sigma / d t$ on photon laboratory energy in shown in Fig. 3. There is a $20 \%$ overall normalization uncertainty, and the data shown in Figs. 2] and 3 have been multiplied by 0.8 .

The spin dependent observables are defined according to Ref. [10]. One set involves both photon and proton helicities. The correlation $K_{L L}$ between the helicities of the incoming photon and the outgoing proton is

$$
K_{L L} \frac{d \sigma}{d t}=\frac{d \sigma\left(\mu=+, \lambda^{\prime}=+\right)}{d t}-\frac{d \sigma\left(\mu=+, \lambda^{\prime}=-\right)}{d t}
$$

and is especially interesting because the experimental result 1] will be announced soon. The correlation, $A_{L L}$, between the incident photon and the proton in the initial state is

$$
A_{L L} \frac{d \sigma}{d t}=\frac{1}{2}\left[\frac{d \sigma(\mu=+, \lambda=+)}{d t}-\frac{d \sigma(\mu=+, \lambda=-)}{d t}\right] .
$$

In the handbag approach of Refs. [8, 10], the amplitudes $\Phi_{--++} \equiv \Phi_{2}$ and $\Phi_{-++-} \equiv \Phi_{6}$ have the same magnitude, and this leads to the prediction that $K_{L L}=A_{L L}$. Our results, for the photon energies of immediate experimental interest $(3.2$ and $4.3 \mathrm{GeV})$ [1, 2], are displayed in Fig. 4, and the related cross sections can be found in Fig. 5. Examining Fig. 4, shows 


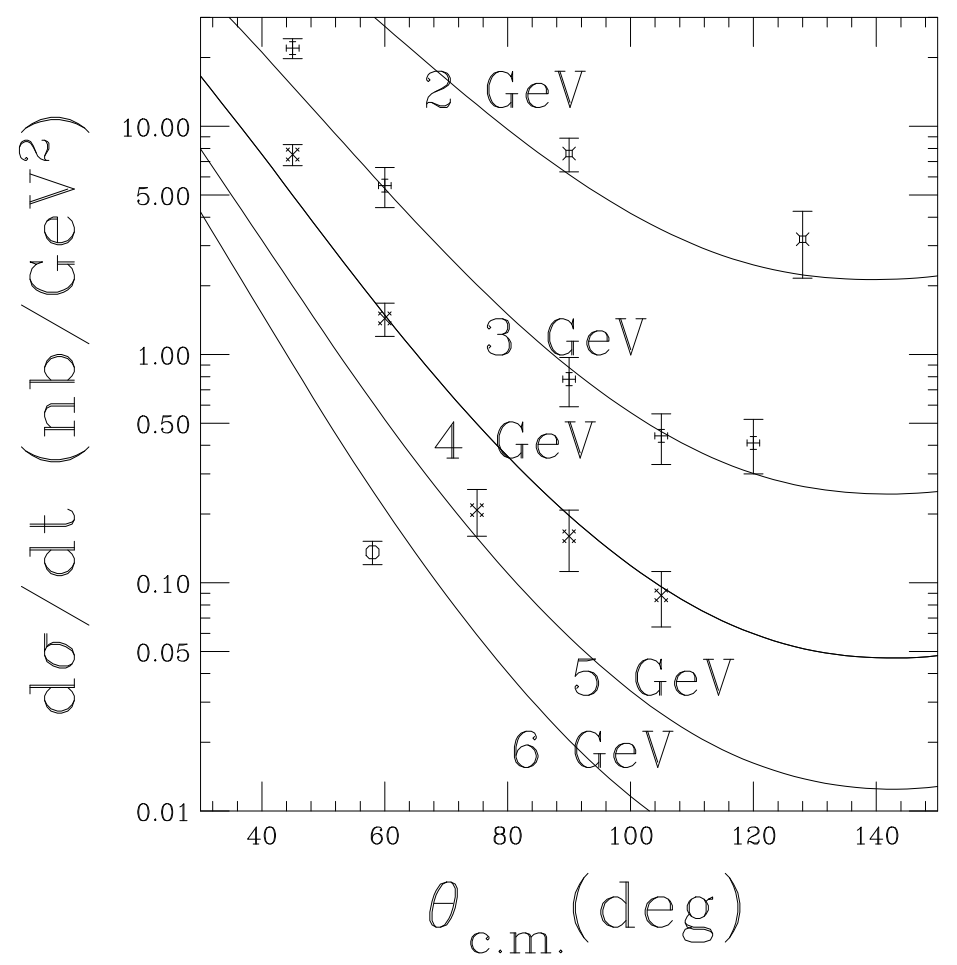

FIG. 3: Energy dependence of cross sections. The data are from Ref. 18]. The curves are obtained using set 3 of Table I, with an increase in the value of $m$ by $10 \%$.

that the predicted values of $K_{L L}$ and $A_{L L}$ do not depend on the quark mass, and have little variation with energy. The values of $K_{L L}$ are large and positive at large scattering angles. This is similar to the $K_{L L}$ of Refs. [8, 10], But their predicted equality between $K_{L L}$ and $A_{L L}$ does not hold here- for backward scattering angles, we find $K_{L L} \approx-A_{L L}$.

This demands explanation. In Refs. 8, 10] the equality between $K_{L L}$ and $A_{L L}$ arises from neglecting the variation of the quark's minus and tranverse momenta, and the effects of quark orbital angular momentum that lead to vanishing proton helicity flip matrix elements. The massless quarks are taken to move collinearly with the proton which consequently does not change helicity. In our model, the role of orbital angular momentum and non-conservation of the proton helicity is the crucial aspect in reproducing the proton form factors 11].

Let's consider scattering by $180^{\circ}$ (back-angle scattering) to illustrate how it is that $K_{L L} \neq A_{L L}$. Examine Eq. (4). The transformation matrices $T(\mathbf{p})_{S \lambda}$ reduce to overlaps between two-component spinors, in which $\boldsymbol{\sigma} \cdot \widehat{\mathbf{p}}|\lambda\rangle=\lambda|\lambda\rangle$. Then for back-angle scattering $T^{*}\left(\mathbf{p}^{\prime}\right)_{S^{\prime} \lambda^{\prime}}=\frac{1}{\sqrt{2}}\left(\delta_{S^{\prime},+1 / 2}+\lambda^{\prime} \delta_{S^{\prime},-1 / 2}\right), T(\mathbf{p})_{S \lambda}=\frac{1}{\sqrt{2}}\left(\delta_{S,+1 / 2}-\lambda \delta_{S,-1 / 2}\right)$, so that $\Phi_{2}=\frac{1}{2}\left[\mathcal{M}_{++}+\mathcal{M}_{--}-\mathcal{M}_{+-}-\mathcal{M}_{-+}\right], \Phi_{6}=\frac{1}{2}\left[\mathcal{M}_{++}+\mathcal{M}_{--}+\mathcal{M}_{+-}+\mathcal{M}_{-+}\right]$. The dependence of $\mathcal{M}_{S^{\prime} S}$ on the photon polarization vectors is suppressed, as these are the same for $\Phi_{2,6}$. Equality of $\Phi_{2}$ and $\Phi_{6}$ can only occur if each of the proton spin flip matrix elements $\mathcal{M}_{+-}, \mathcal{M}_{-+}$vanish, or if their sum vanishes. Inspection of Eq. (3) shows that the spin-flip matrix elements do not vanish, and that the terms $\gamma \cdot\left(q+q^{\prime}\right)=\gamma^{0} 2 q^{0}$ lead to operators $\left(1+\sigma_{x}\right)$ evaluated between Pauli spinors. The operator $\sigma_{x}$ raises and lowers spins with exactly strength, so that $\mathcal{M}_{+-}+\mathcal{M}_{-+}$does not vanish, $\Phi_{2} \neq \Phi_{6}$, and $A_{L L} \neq K_{L L}$.

Another way to understand this inequality is to examine the numerical effect of reducing the quark mass towards 0 . We find that this causes $A_{L L}$ to aproach $K_{L L}$.

There are other polarization variables 10]. The helicity transfer from the incoming to the outgoing photon is given by $D_{L L} d \sigma / d t=\left(d \sigma\left(\mu=+, \mu^{\prime}=+\right) / d t-d \sigma\left(\mu=+, \mu^{\prime}=-\right) / d t\right)$. 


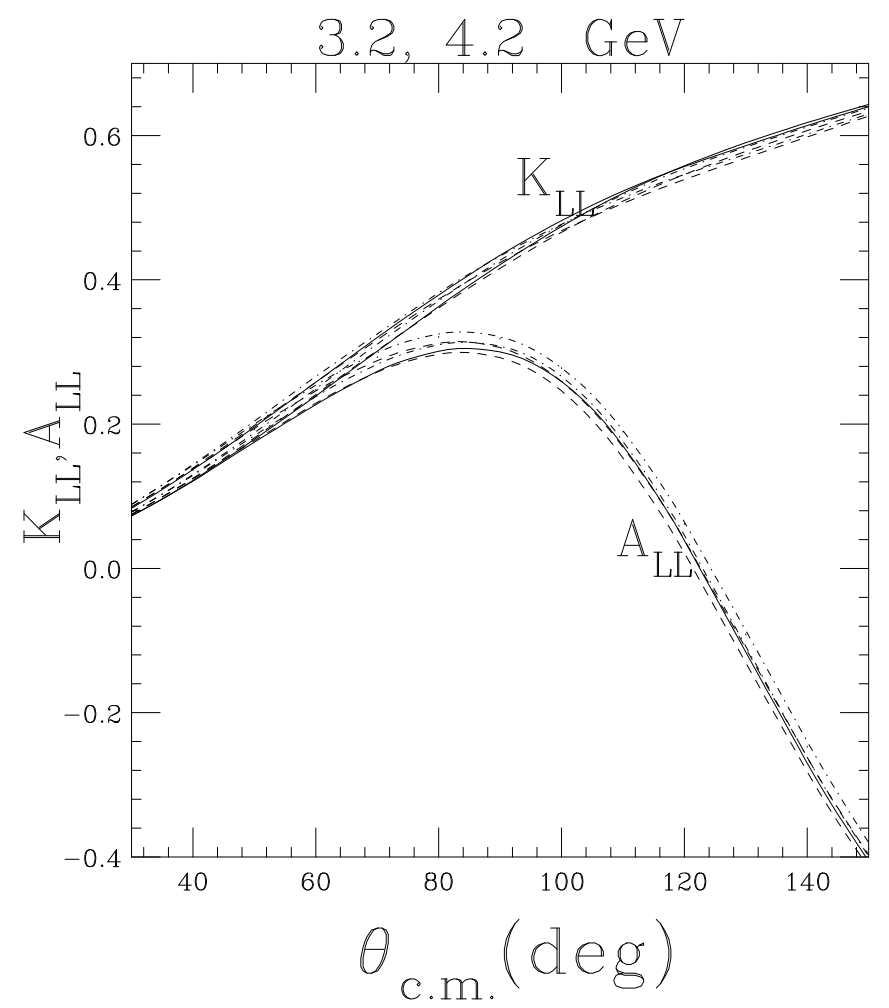

FIG. 4: $K_{L L}, A_{L L}$ obtained using the parameter sets of Fig. 2. These are nearly independent of energy and quark mass.

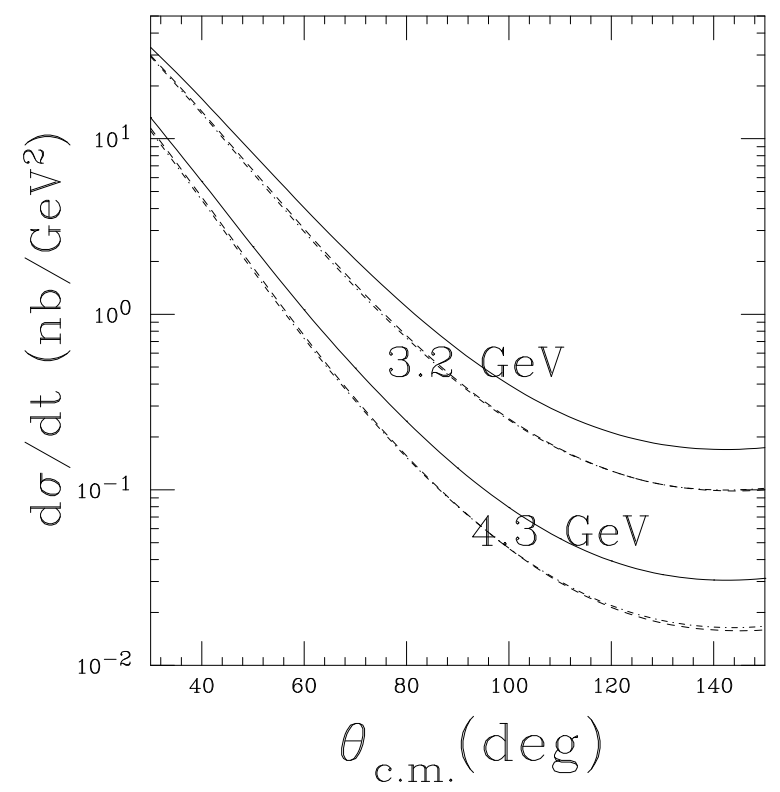

FIG. 5: Computed values of $\frac{d \sigma}{d t}$ the parameter sets of Fig. 2 with an increased quark mass.

Ref. [10] finds that $D_{L L} \approx 1$. The polarization of the incoming proton is defined by $P d \sigma / d t=$ $\frac{1}{2}[d \sigma(\uparrow) / d t-d \sigma(\downarrow) / d t]$. In Ref. [10] small corrections lead to estimating that $P \approx 3 \%$. Our result is that $P=0$. Our prediction for $D_{L L}$, shown in Fig [6, is that it has significant deviations from unity at large scattering angles.

Now consider sideways proton spin directions. The correlation between the helicity of the 


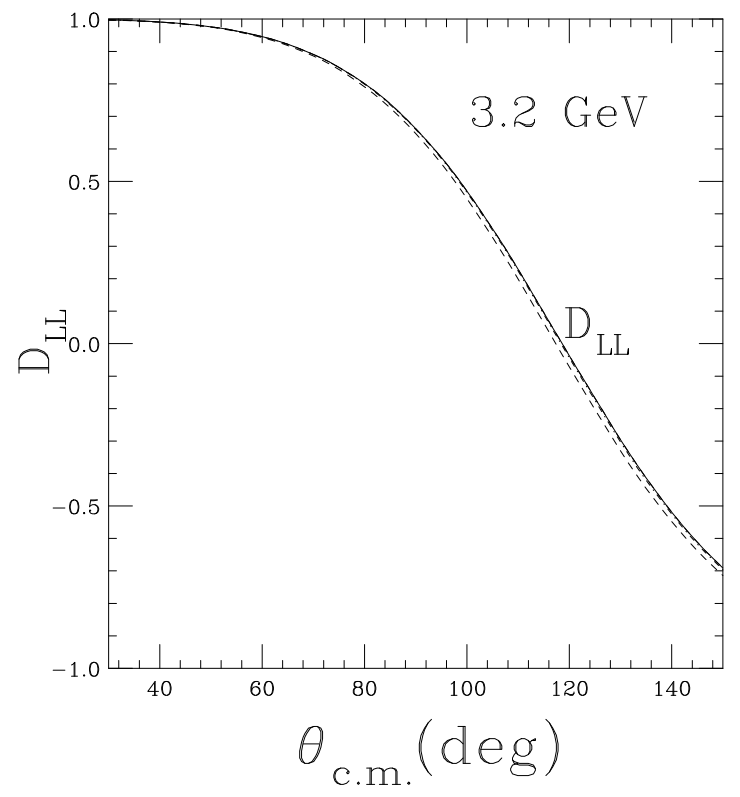

FIG. 6: Computed values of $D_{L L}$, using parameter sets of Table I.

incoming photon and the sideways $(S)$ polarization of the incoming proton, parallel or antiparallel to the $S$-direction is defined [10] as $A_{L S}$, and the one for the sideways polarization of the outgoing proton is $K_{L S}$. We find $K_{L S}=0$ and $A_{L S}=0$, and that the incoming photon asymmetry $\Sigma[10]$ vanishes.

Let's summarize. Poincaré invariance, gauge invariance, conservation of parity and time reversal invariance are respected in our impulse approximation evaluation of the handbag diagrams. Proton wave functions, previously constrained by comparison with measured form factors, that incorporate the influence of quark orbital angular momentum (and the corresponding violation of proton helicity conservation) are used. Computed cross sections are in reasonably good agreement with early measurements. The value of $K_{L L}$ is large and positive for scattering at large angles. In contrast with earlier work, we find that $K_{L L} \neq A_{L L}$, and $D_{L L} \neq 1$ at large scattering angles. With our model functions, photon laboratory energies of $6 \mathrm{GeV}$ or less are too low for the simplifying assumptions that lead to proton helicity conservation to be valid. Future experiments that measure $A_{L L}$ or $D_{L L}$ can determine whether or not proton helicity conservation holds in Compton scattering at any given energy.

\section{Acknowledgments}

I thank B. Wojtsekhowski for suggesting this project, and P. Kroll for useful discussions. I am grateful to the ECT*, the National Institute for Nuclear Theory at the UW, the NSTG at LBL and the CSSSM for providing hospitality during the course of this work. The Guggenheim foundation, on the other hand, was of no help at all. This work is partially 
supported by the USDOE.

[1] Jefferson Laboratory Experiment E99-114, C. Hyde-Wright, A. M. Nathan, and B. Wojtsekhowski, spokespersons.

[2] Jefferson Laboratory Experiment E03-003, A. M. Nathan and B. Wojtsekhowski, spokespersons.

[3] M. K. Jones et al. [Jefferson Lab Hall A Collaboration], Phys. Rev. Lett. 84, 1398 (2000); O. Gayou et al.Phys. Rev. Lett. 88, 092301 (2002)

[4] A. S. Kronfeld and B. Nizic, Phys. Rev. D 44, 3445 (1991) [Erratum-ibid. D 46, 2272 (1992)].

[5] G. R. Farrar and H. y. Zhang, Phys. Rev. Lett. 65, 1721 (1990); Phys. Rev. D 41, 3348 (1990) [Erratum-ibid. D 42, 2413 (1990)]. E. Maina and G. R. Farrar, Phys. Lett. B 206, 120 (1988).

[6] T. C. Brooks and L. J. Dixon, Phys. Rev. D 62, 114021 (2000).

[7] A. V. Radyushkin, Phys. Rev. D 58, 114008 (1998).

[8] M. Diehl, T. Feldmann, R. Jakob and P. Kroll, Eur. Phys. J. C 8, 409 (1999); Phys. Lett. B460,204 (1999)

[9] M. Diehl, Phys. Rept. 388, 41 (2003).

[10] H. W. Huang, P. Kroll and T. Morii, Eur. Phys. J. C 23, 301 (2002) [Erratum-ibid. C 31, 279 (2003)].

[11] G. A. Miller and M. R. Frank, Phys. Rev. C 65, 065205 (2002)

[12] M.R. Frank, B.K. Jennings and G.A. Miller, Phys. Rev. C 54, 920 (1996).

[13] G. A. Miller, Phys. Rev. C 66, 032201 (2002).

[14] V. B. Berestetskii and M. V. Terent'ev. Sov. J. Nucl. Phys. 25, 347 (1977).

[15] P. L. Chung and F. Coester. Phys. Rev. D 44, 229, (1991); F. Coester, Prog. in Part. and Nucl. Phys. 29, 1 (1992). F. Schlumpf, U. Zurich Ph. D. Thesis, hep-ph/9211255.

[16] S. Théberge, and G. A. Miller, Phys. Rev. D24 (1981) 216; S. Théberge, G. A. Miller and A. W. Thomas, Can. J. Phys. 60, 59 (1982). G. A. Miller, A. W. Thomas and S. Théberge, Phys. Lett. B 91, 192 (1980).

[17] H. Rollnik and P. Stichel, in E.Paul et al., Elementary Particle Physics, Springer Tracts Vol.79, Berlin 1976. ; C. Bourrely, J. Soffer and E. Leader, Phys. Rept. 59, 95 (1980). M. Diehl, Eur. Phys. J. C 19, 485 (2001).

[18] M. A. Shupe et al., Phys. Rev. D 19, 1921 (1979). 